\title{
CONSTRUCTIONS OF FREE COMMUTATIVE INTEGRO-DIFFERENTIAL ALGEBRAS
}

\author{
XING GAO AND LI GUO
}

\begin{abstract}
AвSTRACT. In this survey, we outline two recent constructions of free commutative integro-differential algebras. They are based on the construction of free commutative Rota-Baxter algebras by mixable shuffles. The first is by evaluations. The second is by the method of Gröbner-Shirshov bases.
\end{abstract}

\section{Contents}

1. Introduction

2. Definitions and preliminary constructions

2.1. The definitions

2.2. Free differential Rota-Baxter algebras

2.3. Free commutative operated algebras

3. Free commutative integro-differential algebras by initialization

3.1. Regular differential algebras

3.2. Construction of $\operatorname{ID}(A)^{*}$

4. Free commutative integro-differential algebras by Gröbner-Shirshov bases 11

4.1. Weakly monomial order 12

4.2. Composition-Diamond lemma

4.3. Free commutative integro-differential algebras by Gröbner-Shirshov bases

References

\section{INTRODUCTION}

In this survey article, we give an outline of the recent constructions of free commutative integrodifferential algebras.

The main axiom of integro-differential algebra can be regarded as an algebraic abstraction of the integral by parts formula which involves both derivation and integration. Thus to understand this abstraction better, we first review the abstraction for derivation and for integration.

In this paper, by an algebra we mean a commutative associative algebra over some commutative ring, unless otherwise specified. A differential algebra is an algebra $R$ together with a linear operator $d: R \rightarrow R$ that satisfies the following axiom distilled from the Leibniz rule for derivations

$$
d(x y)=d(x) y+x d(y), \text { for all } x, y \in R .
$$

Date: September 29, 2017.

2010 Mathematics Subject Classification. 16S15, 13P10, 16W99, 12H05, 47G20 .

Key words and phrases. Differential algebra, Rota-Baxter algebra, integro-differential algebra, Gröbner-Shirshov basis, free algebra, shuffle product, mixable shuffle product. 
The study of differential algebra began with Ritt's classic work [35, 36]. After the fundamental work of Kolchin [33], differential algebra has evolved into a vast area of mathematics that is important in both theory [15, 44] and applications: for instance, in mechanic theorem proving by W.-T. Wu [45, 46]. Free (commutative) differential algebras, in the form of differential polynomial algebras (Theorem 2.3), are essential for studying differential equations, as polynomial algebras are for commutative algebras.

The algebraic study of integrals came much later. In fact the development did not start from an algebraic abstraction of integrals, but from the effort of G. Baxter [5] in 1960 to understand a formula in probability theory. As a result, the concept is not called an integral algebra, but called a (Rota-)Baxter algebra (Eq. (B) which is the integral counterpart of the derivation, the difference operator, and divided differences (see Eq. (四). Soon afterwards Rota noticed its importance in combinatorics and promoted its study through research and survey articles (see e.g. [40, 41]). Independently, Rota-Baxter operators on Lie algebras were found to be closely related to the classical Yang-Baxter equation [42]. Since the turn of this century, the theory of Rota-Baxter algebra has experienced rapid development with broad applications in mathematics and physics [ $4,21,29,40,41,42$ ], especially noteworthy in the Hopf algebra approach of ConnesKreimer to renormalization of quantum field theory [16, 19, 29]. Here again a fundamental role is played by free (commutative) Rota-Baxter algebras that were first constructed by Rota [40] and Cartier [14], and then by Guo-Keigher [24] in terms of mixable shuffles (Theorem 2.4).

The fusion of differential and Rota-Baxter algebras, motivated by algebraic study of calculus as a whole, appeared about five years ago. It is amazing that two structures for this purpose were introduced at about the same time. One is a relatively simple coupling of differential algebra and Rota-Baxter algebra through section axiom (Eq. (円) that reflects the First Fundamental Theorem of Calculus. It is called differential Rota-Baxter algebra [26]. The other one is a more faithful abstraction of the integration-by-parts formula (see Eq. (7)), giving rise to the concept of an integro-differential algebra [37] which has generated much interest [1], 2, 38, 39]. As suggested in previous cases, free objects for these algebraic structures are important in their studies. Because of the relative independence of the differential and integral (Rota-Baxter) structures in a differential Rota-Baxter algebra, the free object was constructed by a clear combination of the free objects on the differential and Rota-Baxter sides and were obtained at the same time when the concept was introduced. In contrast, the construction of free integro-differential algebras took longer to achieve. Nevertheless, there are two recent constructions [23, 27] and it is the purpose of this paper to give the preliminary background and a summary of these constructions.

Both are based on the construction of free Rota-Baxter algebras by mixable shuffles. Straight from the definition, a free commutative integro-differential algebra can be obtained as the quotient of a free Rota-Baxter algebra modulo the integral-by-parts axiom. By an explicit construction of a free integro-differential algebra, we mean identifying a specific vector space basis of this quotient. Thus we give two such bases in this paper.

After a preliminary Section 2 on the concepts of differential, Rota-Baxter and differential RotaBaxter algebras as well as operated algebras, and the constructions of their respective free objects, we give the first construction [27] of free integro-differential algebras in Section 3. This construction applies to regular differential algebras, a concept which we also review in Section 3. Common examples of regular differential algebras include differential polynomial algebras and rational functions. The second construction [23] is given in Section 4. The construction applies the general method of Gröbner-Shirshov bases, of which Gröbner bases in commutative algebra are special cases, but which apply to many other algebraic structures. We give some details of the 
method in the case of integro-differential algebras, where we use the ambient algebraic structure of a free Rota-Baxter algebra to establish the Composition-Diamond Lemma.

\section{DeFinITIONS AND PRELIMINARY CONSTRUCTIONS}

We recall the definitions of algebras with various differential and integral operators and the constructions of the free objects in the corresponding categories. Free commutative integrodifferential algebras, which are the focus of this survey, will be discussed in later sections.

2.1. The definitions. We recall the algebraic structures considered in this paper. We also introduce variations with nilpotent derivation that will be needed later. Algebras considered in this paper are assumed to be unitary (and commutative), unless explicitly designated as non-unitary.

Definition 2.1. Let $\mathbf{k}$ be a unitary commutative ring. Let $\lambda \in \mathbf{k}$ be fixed.

(a) A differential k-algebra of weight $\lambda$ (also called a $\lambda$-differential k-algebra) is an associative k-algebra $R$ together with a linear operator $d: R \rightarrow R$ such that

$$
d(x y)=d(x) y+x d(y)+\lambda d(x) d(y) \text { for all } x, y \in R,
$$

and

$$
d(1)=0 .
$$

Such an operator is called a derivation of weight $\lambda$ or a $\lambda$-derivation.

(b) A Rota-Baxter k-algebra of weight $\lambda$ is an associative $\mathbf{k}$-algebra $R$ together with a linear operator $P: R \rightarrow R$ such that

$$
P(u) P(v)=P(u P(v))+P(P(u) v)+\lambda P(u v) \quad \text { for all } u, v \in R .
$$

(c) A differential Rota-Baxter k-algebra of weight $\lambda$ (also called a $\lambda$-differential RotaBaxter k-algebra) is a differential k-algebra $(R, d)$ of weight $\lambda$ with a Rota-Baxter operator $P$ of weight $\lambda$ such that

$$
d \circ P=\text { id. }
$$

(d) An integro-differential k-algebra of weight $\lambda$ (also called a $\lambda$-integro-differential kalgebra) is a differential k-algebra $(R, D)$ of weight $\lambda$ with a linear operator $\Pi: R \rightarrow R$ such that

$$
D \circ \Pi=\mathrm{id}_{R}
$$

and

$$
\Pi(D(x)) \Pi(D(y))=\Pi(D(x)) y+x \Pi(D(y))-\Pi(D(x y)) \quad \text { for all } x, y \in R .
$$

Eqs. (3), (5) and (6) are called the Rota-Baxter axiom, section axiom and hybrid RotaBaxter axiom, respectively. It is proved in [27] that a differential k-algebra $(R, D)$ with a linear operator $\Pi: R \rightarrow R$ is an integro-differential algebra if and only if Eq. (5) and the following integration by parts axioms hold:

$$
x \Pi(y)=\Pi(D(x) \Pi(y))+\Pi(x y)+\lambda \Pi(D(x) y)
$$

and

$$
\Pi(x) y=\Pi(\Pi(x) D(y))+\Pi(x y)+\lambda \Pi(x D(y)) \text { for all } x, y \in R .
$$

These two equations can be regarded as the weighed and noncommutative versions of the classical integration by parts formula in analysis. 
Example 2.2. Let $R=C^{\infty}(\mathbb{R})$

(a) Fix a $\lambda \in \mathbb{R}$. Define

$$
D_{\lambda}: R \longrightarrow R, \quad f(x) \mapsto \frac{f(x+\lambda)-f(x)}{\lambda} .
$$

Then $D_{\lambda}$ is a differential operator of weight $\lambda$.

(b) For fixed $a \in \mathbb{R}$, the integral operator

$$
\Pi: R \longrightarrow R, \quad f(x) \mapsto \int_{a}^{x} f(t) d t
$$

is a Rota-Baxter operator of weight zero.

(c) $D$ be the usual derivation on $R$ and $\Pi$ be the above integral operator. Then $(R, D, \Pi)$ is a differential Rota-Baxter algebra and an integro-differential algebra of weight 0 .

See [21, 26, 27, 41] for more examples.

2.2. Free differential Rota-Baxter algebras. We first recall the construction of free commutative differential algebras and introduce their order $n$ variations. For a set $Y$, let $C(Y)$ denote the free commutative monoid on $Y$. Thus elements in $C(Y)$ are commutative words from the alphabet set $Y$, plus the identity 1 . Let $\mathbf{k}[Y]$ be the commutative polynomial algebra generated by $Y$.

Theorem 2.3. ([23, 26])

(a) Let $Y$ be a set with a map $d_{0}: Y \rightarrow \mathbf{k}[Y]$. Extend $d_{0}$ to $d: \mathbf{k}[Y] \rightarrow \mathbf{k}[Y]$ as follows. Let $w=u_{1} \cdots u_{k}$, where $u_{i} \in Y$ for $1 \leq i \leq k$, be a commutative word from the alphabet set $Y$. Recursively define

$$
d(w)=d_{0}\left(u_{1}\right) u_{2} \cdots u_{k}+u_{1} d\left(u_{2} \cdots u_{k}\right)+\lambda d_{0}\left(u_{1}\right) d\left(u_{2} \cdots u_{k}\right) .
$$

Explicitly,

$$
d(w)=\sum_{\emptyset \neq I \subseteq\{1, \cdots, k\}} \lambda^{|I|-1} \check{d}_{I}\left(u_{1}\right) \cdots \check{d}_{I}\left(u_{k}\right), \text { where } \check{d}_{I}\left(u_{i}\right)= \begin{cases}d_{0}\left(u_{i}\right), & i \in I, \\ u_{i}, & i \notin I .\end{cases}
$$

Further define $d(1)=0$ and then extend $d$ to $\mathbf{k}[Y]$ by linearity. Then $(\mathbf{k}[Y], d)$ is a differential algebra of weight $\lambda$.

(b) Let $X$ be a set. Let $Y:=\Delta X:=\left\{x^{(n)} \mid x \in X, n \geq 0\right\}$ with the map $d_{0}: \Delta X \rightarrow \Delta X, x^{(n)} \mapsto$ $x^{(n+1)}$. Then with the extension $d_{X}:=d$ of $d_{0}$ as in $E q$. (Q), $\left(\mathbf{k}\{X\}, d_{X}\right):=\left(\mathbf{k}[\Delta X], d_{X}\right)$ is the free commutative differential algebra of weight $\lambda$ on the set $X$.

(c) For a given $n \geq 1$, let $\Delta X^{(n+1)}:=\left\{x^{(k)} \mid x \in X, k \geq n+1\right\}$. Then $\mathbf{k}\{X\} \Delta X^{(n+1)}$ is the differential ideal $I_{n}$ of $\mathbf{k}\{X\}$ generated by the set $\left\{x^{(n+1)} \mid x \in X\right\}$. The quotient differential algebra $\mathbf{k}\{X\} / I_{n}$ has a canonical basis given by $\Delta_{n} X:=\left\{x^{(k)} \mid k \leq n\right\}$, thus giving a differential algebra isomorphism $\mathbf{k}\{X\} / I_{n} \cong \mathbf{k}\left[\Delta_{n} X\right]$ where the differential structure on the later algebra is given by $d$ in Eq. (19), where

$$
d_{0}\left(x^{(i)}\right)= \begin{cases}x^{(i+1)}, & 1 \leq i \leq n-1 \\ 0, & i=n\end{cases}
$$

We note that in $\mathbf{k}\left[\Delta_{n} X\right], d_{0}^{n+1}(u)=0$ only holds for the variables $x \in X$. For example, when $n=1$, we have $d_{0}^{2}\left(x^{2}\right)=2 x^{(1)} \neq 0$. 
We next recall the construction of free commutative Rota-Baxter algebras in terms of mixable shuffles [24, 25]. The mixable shuffle product is shown to be the same as the quasi-shuffle product of Hoffman [18, 29, 32]. Let $A$ be a commutative k-algebra. Define

$$
\amalg(A)=\bigoplus_{k \geq 0} A^{\otimes(k+1)}=A \oplus A^{\otimes 2} \oplus \cdots .
$$

Let $\mathfrak{a}=a_{0} \otimes \cdots \otimes a_{m} \in A^{\otimes(m+1)}$ and $\mathfrak{b}=b_{0} \otimes \cdots \otimes b_{n} \in A^{\otimes(n+1)}$. If $m=0$ or $n=0$, define

$$
\mathfrak{a} \diamond \mathfrak{b}= \begin{cases}\left(a_{0} b_{0}\right) \otimes b_{1} \otimes \cdots \otimes b_{n}, & m=0, n>0, \\ \left(a_{0} b_{0}\right) \otimes a_{1} \otimes \cdots \otimes a_{m}, & m>0, n=0, \\ a_{0} b_{0}, & m=n=0 .\end{cases}
$$

If $m>0$ and $n>0$, inductively (on $m+n$ ) define

$$
\begin{aligned}
\mathfrak{a} \diamond \mathfrak{b}=\left(a_{0} b_{0}\right) \otimes & \left(a_{1} \otimes a_{2} \otimes \cdots \otimes a_{m}\right) \diamond\left(1 \otimes b_{1} \otimes \cdots \otimes b_{n}\right) \\
& +\left(1 \otimes a_{1} \otimes \cdots \otimes a_{m}\right) \diamond\left(b_{1} \otimes \cdots \otimes b_{n}\right) \\
& \left.+\lambda\left(a_{1} \otimes \cdots \otimes a_{m}\right) \diamond\left(b_{1} \otimes \cdots \otimes b_{n}\right)\right) .
\end{aligned}
$$

Extending by additivity, we obtain a k-bilinear map

$$
\diamond: \amalg(A) \times \amalg(A) \rightarrow \amalg(A) .
$$

Alternatively,

$$
\mathfrak{a} \diamond \mathfrak{b}=\left(a_{0} b_{0}\right) \otimes\left(\overline{\mathfrak{a}}_{\amalg \lambda} \overline{\mathfrak{b}}\right)
$$

where $\overline{\mathfrak{a}}=a_{1} \otimes \cdots \otimes a_{m}, \overline{\mathfrak{b}}=b_{1} \otimes \cdots \otimes b_{n}$ and ${ }_{\omega_{\lambda}}$ is the mixable shuffle (quasi-shuffle) product of weight $\lambda$ [21, 24, 32], which specializes to the shuffle product $\amalg$ when $\lambda=0$.

Define a k-linear endomorphism $P_{A}$ on $\amalg(A)$ by assigning

$$
P_{A}\left(x_{0} \otimes x_{1} \otimes \cdots \otimes x_{n}\right)=1_{A} \otimes x_{0} \otimes x_{1} \otimes \cdots \otimes x_{n},
$$

for all $x_{0} \otimes x_{1} \otimes \cdots \otimes x_{n} \in A^{\otimes(n+1)}$ and extending by additivity. Let $j_{A}: A \rightarrow \amalg(A)$ be the canonical inclusion map.

Theorem 2.4. (24, 25]) Let A be a commutative k-algebra.

(a) The pair $\left(\amalg(A), P_{A}\right)$, together with the natural embedding $j_{A}: A \hookrightarrow \amalg(A)$, is the free commutative Rota-Baxter $\mathbf{k}$-algebra on A of weight $\lambda$. In other words, for any commutative Rota-Baxter k-algebra $(R, P)$ and any k-algebra map $\varphi: A \rightarrow R$, there exists a unique Rota-Baxter k-algebra homomorphism $\tilde{\varphi}:\left(\amalg(A), P_{A}\right) \rightarrow(R, P)$ such that $\varphi=\tilde{\varphi} \circ j_{A}$ as k-algebra homomorphisms.

(b) Let $Y$ be a set and let $\mathbf{k}[Y]$ be the free commutative algebra on $Y$. The pair $\left(\amalg(Y), P_{Y}\right):=$ $\left(\amalg(\mathbf{k}[Y]), P_{\mathbf{k}[Y]}\right)$, together with the natural embedding $j_{Y}: Y \hookrightarrow \mathbf{k}[Y] \rightarrow \amalg(\mathbf{k}[Y])$, is the free commutative Rota-Baxter $\mathbf{k}$-algebra of weight $\lambda$ on $Y$.

Since $\diamond$ is compatible with the multiplication in $A$, we will often suppress the symbol $\diamond$ and simply write $x y$ for $x \diamond y$ in $\amalg(A)$, unless there is a danger of confusion.

A linear basis of $\amalg(\mathbf{k}[Y])$ is given by

$$
\mathcal{B}(Y):=\left\{x_{0} \otimes \cdots \otimes x_{k} \mid x_{i} \in C(Y), 0 \leq i \leq k, k \geq 0\right\},
$$

called the set of Rota-Baxter monomials in $Y$. The integer $\operatorname{dep}\left(x_{0} \otimes \cdots \otimes x_{k}\right):=k+1$ is called the depth of $x_{0} \otimes \cdots \otimes x_{k}$. As a convenience, we also write $P$ for $P_{\mathbf{k}[Y]}$. Then $1 \otimes u$ and $P(u)$ stand for the same element, and we will use both notations synonymously in this paper. 
We now put the differential and Rota-Baxter algebra structures together. Let $(A, d)$ be a commutative differential k-algebra of weight $\lambda$. Extend $d$ to $\amalg(A)$ by

$$
\begin{aligned}
& d_{A}\left(u_{0} \otimes u_{1} \otimes \cdots \otimes u_{k}\right) \\
& \quad=d\left(u_{0}\right) \otimes u_{1} \otimes \cdots \otimes u_{k}+u_{0} u_{1} \otimes u_{2} \otimes \cdots \otimes u_{k}+\lambda d\left(u_{0}\right) u_{1} \otimes u_{2} \otimes \cdots \otimes u_{k}, \quad k \geq 0 .
\end{aligned}
$$

Note that $d_{A}$ does not satisfy the Leibniz rule with respect to the tensor product since here a tensor factor means an application of the Rota-Baxter operator $P_{A}: u_{0} \otimes u_{1}=u_{0} P_{A}\left(u_{1}\right)$. Thus

$d_{A}\left(u_{0} \otimes u_{1}\right)=d_{A}\left(u_{0}\right) P_{A}\left(u_{1}\right)+u_{0} d_{A}\left(P_{A}\left(u_{1}\right)\right)+\lambda d_{A}\left(u_{0}\right) d_{A}\left(P_{A}\left(u_{1}\right)\right)=d\left(u_{0}\right) \otimes u_{1}+u_{0} u_{1}+\lambda d\left(u_{0}\right) u_{1}$.

Theorem 2.5. ([23, 26]) Let $Y$ be a set with a set map $d_{0}: Y \rightarrow \mathbf{k}[Y]$ and let $(\mathbf{k}[Y], d)$ be the commutative differential algebra of weight $\lambda$ in Theorem 2.3. (a). The triple $\left(\amalg(\mathbf{k}[Y]), d_{\mathbf{k}[Y]}, P_{\mathbf{k}[Y]}\right)$, together with $j_{\mathbf{k}[Y]}: \mathbf{k}[Y] \rightarrow \amalg(\mathbf{k}[Y])$, is the free commutative differential Rota-Baxter $\mathbf{k}$-algebra of weight $\lambda$ on the differential algebra $(\mathbf{k}[Y], d)$.

Apply Theorem 2.5 to $Y:=\Delta X$ and $d_{0}$ as in Theorem 2.3.(b). From Eq. 14, the set

$$
\mathcal{B}(\Delta X):=\left\{u_{0} \otimes \cdots \otimes u_{k} \mid u_{i} \in C(\Delta X), 0 \leq i \leq k, k \geq 0\right\}
$$

is a $\mathbf{k}$-basis of the free commutative differential Rota-Baxter algebra $\amalg(\Delta X)$ on the free differential algebra $\left(\mathbf{k}\{X\}, d_{X}\right)$. We call this basis the set of differential Rota-Baxter (DRB) monomials on $X$. Similarly, for $n \geq 1$, apply Theorem 2.5 to $Y:=\Delta_{n} X$ and $d_{0}$ as in Eq. (10) of Theorem 2.3. (c). Then $\mathcal{B}\left(\Delta_{n} X\right)$ is a basis of $\amalg\left(\Delta_{n} X\right)$ and is called the set of DRB monomials of order $n$ on $X$.

2.3. Free commutative operated algebras. The construction of the free commutative operated algebra on a set $X$ that has the free commutative (differential) Rota-Baxter algebra as a quotient is given in [23]. The explicit construction $\amalg(X)$ of the free commutative Rota-Baxter algebra in Theorem 2.4 can be realized on a submodule of the free commutative operated algebra spanned by reduced words under a rewriting rule defined by the Rota-Baxter axiom.

This construction is parallel to that of the free (noncommutative) operated algebra on a set in [11, 20, 21, 28]. See [34] for the non-unitary case.

Definition 2.6. Let $\Omega$ be a set. A commutative $\Omega$-operated monoid is a commutative monoid $G$ together with maps $\alpha_{\omega}: G \rightarrow G, \omega \in \Omega$. A homomorphism between commutative $\Omega$-operated monoids $\left(G,\left\{\alpha_{\omega}\right\}_{\omega}\right)$ and $\left(H,\left\{\beta_{\omega}\right\}_{\omega}\right)$ is a monoid homomorphism $f: G \rightarrow H$ such that $f \circ \alpha_{\omega}=\beta_{\omega} \circ f$ for $\omega \in \Omega$.

We similarly define the concept of a commutative $\Omega$-operated $\mathbf{k}$-algebra. The suffix $\Omega$ will be suppressed when the meaning of $\Omega$ is clear from the context. We recall the construction of the free objects in the category of commutative operated monoids [23].

Fix a set $Y$. Define monoids $\mathfrak{C}_{n}:=\mathfrak{C}_{n}(Y)$ for $n \geq 0$ by a recursion. First denote $\mathfrak{C}_{0}:=C(Y)$. For each $\omega \in \Omega$, let $\lfloor C(Y)\rfloor_{\omega}:=\left\{\lfloor u\rfloor_{\omega} \mid u \in C(Y)\right\}$ be a set in bijection with $C(Y)$. We require that all the sets $C(Y)$ and $\lfloor C(Y)\rfloor_{\omega}, \omega \in \Omega$ are disjoint from one another. We write the notation $\sqcup$ for the disjoint union. Then define

$$
\mathfrak{C}_{1}:=C\left(Y \sqcup\left(\sqcup_{\omega \in \Omega}\lfloor C(Y)\rfloor_{\omega}\right)\right)=C\left(Y \sqcup\left(\sqcup_{\omega \in \Omega}\left\lfloor\mathfrak{C}_{0}\right\rfloor_{\omega}\right)\right) .
$$

Note that elements in $\lfloor C(Y)\rfloor_{\omega}$ are only symbols indexed by elements in $C(Y)$. For example, $\lfloor 1\rfloor_{\omega}$ is not the identity, but a new symbol. The inclusion $Y \hookrightarrow Y \sqcup\left(\sqcup_{\omega \in \Omega}\left\lfloor\mathfrak{C}_{0}\right\rfloor_{\omega}\right)$ induces a monomorphism $i_{0}: \mathfrak{C}_{0}=C(Y) \hookrightarrow \mathfrak{C}_{1}=C\left(Y \sqcup\left(\sqcup_{\omega \in \Omega}\left\lfloor\mathfrak{C}_{0}\right\rfloor_{\omega}\right)\right)$ of free commutative monoids through which we 
identify $\mathfrak{C}_{0}$ with its image in $\mathfrak{C}_{1}$. Inductively assume that $\mathfrak{C}_{n-1}$ have been defined for $n \geq 2$ and that the injection

$$
i_{n-2}: \mathfrak{C}_{n-2} \hookrightarrow \mathfrak{C}_{n-1}
$$

has been obtained. Then define

$$
\mathfrak{C}_{n}:=C\left(Y \sqcup\left(\sqcup_{\omega \in \Omega}\left\lfloor\mathfrak{C}_{n-1}\right\rfloor_{\omega}\right)\right)
$$

Also the injection $i_{n-2}$ gives an injection

$$
\left\lfloor\mathfrak{C}_{n-2}\right\rfloor_{\omega} \hookrightarrow\left\lfloor\mathfrak{C}_{n-1}\right\rfloor_{\omega}, \omega \in \Omega .
$$

Thus by the freeness of $\mathfrak{C}_{n-1}=C\left(Y \sqcup\left(\sqcup_{\omega \in \Omega}\left\lfloor\mathfrak{C}_{n-2}\right\rfloor_{\omega}\right)\right)$ as a free commutative monoid, we obtain

$$
i_{n-1}: \mathfrak{C}_{n-1}=C\left(Y \sqcup\left(\sqcup_{\omega \in \Omega}\left\lfloor\mathfrak{C}_{n-2}\right\rfloor_{\omega}\right)\right) \hookrightarrow C\left(Y \sqcup\left(\sqcup_{\omega \in \Omega}\left\lfloor\mathfrak{C}_{n-1}\right\rfloor_{\omega}\right)\right)=\mathfrak{C}_{n} .
$$

Finally, define the commutative monoid

$$
\mathfrak{C}(Y):=\bigcup_{n \geq 0} \mathfrak{c}_{n}=\underset{\lim }{\longrightarrow} \mathfrak{c}_{n} .
$$

Elements in $\mathfrak{C}(Y)$ are called (commutative) $\Omega$-bracketed monomials in $Y$. Defining

$$
\lfloor\rfloor_{\omega}: \mathfrak{C}(Y) \rightarrow \mathfrak{C}(Y), \quad u \mapsto\lfloor u\rfloor_{\omega}, \omega \in \Omega,
$$

then $\left.\left(\mathscr{C}(Y),\{\mathrm{L}\rfloor_{\omega}\right\}_{\omega \in \Omega}\right)$ is a commutative operated monoid and its linear span $\left.\left(\mathbf{k} \mathscr{C}(Y),\{\mathrm{L}\rfloor_{\omega}\right\}_{\omega \in \Omega}\right)$ is a commutative (unitary) $\Omega$-operated k-algebra with its multiplication extended from $\mathfrak{C}(Y)$ by linearity.

Proposition 2.7. ([23]) Let $\Omega$ be a set.

(a) Let $j_{Y}: Y \hookrightarrow \mathfrak{C}(Y)$ be the natural embedding. Then the triple $\left.\left(\mathfrak{C}(Y),\{\mathrm{L}\rfloor_{\omega}\right\}_{\omega \in \Omega}, j_{Y}\right)$ is the free commutative operated monoid on $Y$. More precisely, for any commutative operated monoid $G$ and set map $f: Y \rightarrow G$, there is a unique extension of $f$ to a homomorphism $\bar{f}: \mathfrak{C}(Y) \rightarrow G$ of operated monoids.

(b) Let $j_{Y}: Y \hookrightarrow \mathbf{k} \mathfrak{C}(Y)$ be the natural embedding. Then the triple $\left.\left(\mathbf{k} \mathfrak{C}(Y),\{\mathrm{L}\rfloor_{\omega}\right\}_{\omega \in \Omega}, j_{Y}\right)$ is the free commutative operated unitary $\mathbf{k}$-algebra on $Y$. More precisely, for any commutative $\mathbf{k}$-algebra $R$ and set map $f: Y \rightarrow R$, there is a unique extension of $f$ to a homomorphism $\bar{f}: \mathbf{k} \mathfrak{C}(Y) \rightarrow R$ of operated $\mathbf{k}$-algebras.

By the universal property of $\mathbf{k} \mathfrak{C}(Y)$, the following conclusion from general principles of universal algebra is obtained [3, 17].

Proposition 2.8. ([23]) Let $Y$ be a set with $d_{0}: Y \rightarrow \mathbf{k}[Y]$. Let $\Omega=\{d, P\}$ and write $d(u):=$ $\lfloor u\rfloor_{d}, P(u):=\lfloor u\rfloor_{P}$. Let $I_{D R B}=I_{D R B, Y}$ be the operated ideal of $\mathbf{k} \mathbb{C}(Y)$ generated by the set

$$
\left\{\begin{array}{l|l}
d(r)-d_{0}(r), & \\
d(u v)-d(u) v-u d(v)-\lambda d(u) d(v), & \\
P(u) P(v)-P(u P(v))-P(P(u) v)-\lambda P(u v), & \mid r \in Y, u, v \in \mathbb{C}(Y) \\
(d \circ P)(u)-u
\end{array}\right\} .
$$

Then the quotient operated algebra $\mathbf{k} \subseteq(Y) / I_{D R B}$, with operations induced by $d$ and $P$ (which we again denote by $d$ and $P$, respectively), is the free commutative differential Rota-Baxter algebra on the differential algebra $(\mathbf{k}[Y], d)$ in Theorem 2.3. $[a$ ).

Combining Proposition 2.8 with Theorem 2.5, we have 
Proposition 2.9. ([23]) Let $Y$ be a set with $d_{0}: Y \rightarrow \mathbf{k}[Y]$. The natural embedding $\eta: \amalg(\mathbf{k}[Y]) \hookrightarrow \mathbf{k} C(Y), \quad u_{0} \otimes u_{1} \otimes \cdots \otimes u_{k} \mapsto u_{0} P\left(u_{1} P\left(\cdots P\left(u_{k}\right) \cdots\right)\right), u_{i} \in C(Y), 0 \leq i \leq k, k \geq 0$, composed with the quotient map $\rho:=\rho_{Y}: \mathbf{k} \mathfrak{C}(Y) \rightarrow \mathbf{k} \mathfrak{C}(Y) / I_{D R B}$ gives a linear bijection (in fact, an isomorphism of differential Rota-Baxter algebras)

$$
\theta:=\theta_{Y}: \amalg(\mathbf{k}[Y]) \rightarrow \mathbf{k} \mathfrak{C}(Y) / I_{D R B} .
$$

Because of the bijectivity of $\theta$, we can identify the basis $\mathcal{B}(Y)$ of $\amalg(\mathbf{k}[Y])$ in Eq. (14) with its image $\eta(\mathcal{B}(Y))$ in $\mathbf{k} \mathfrak{C}(Y)$ :

$$
x_{0} \otimes x_{1} \otimes \cdots \otimes x_{k} \leftrightarrow x_{0} P\left(x_{1} P\left(\cdots P\left(x_{k}\right) \cdots\right)\right), x_{i} \in C(Y), 0 \leq i \leq k, k \geq 0 .
$$

Define the reduction map

$$
\operatorname{Red}:=\operatorname{Red}_{Y}:=\theta^{-1} \circ \rho: \mathbf{k} \mathfrak{C}(Y) \rightarrow \mathbf{k} \mathfrak{C}(Y) / I_{D R B, Y} \rightarrow \amalg(\mathbf{k}[Y]) \cong \eta(\amalg(\mathbf{k}[Y])) .
$$

It reduces any $(d, P)$-bracketed monomial on $Y$ to a linear combination of DRB monomials on $Y$. For example, if $u, v \in C(Y)$, then

$\operatorname{Red}\left(\lfloor u\rfloor_{P}\lfloor v\rfloor_{P}\right)=\operatorname{Red}(P(u) P(v))=1 \otimes u \otimes v+1 \otimes v \otimes u+\lambda \otimes u v \leftrightarrow P(u P(v))+P(v P(u))+\lambda P(u v)$.

\section{FreE COMMUTATIVE INTEGRO-DIFFERENTIAL ALGEBRAS BY INITIALIZATION}

In this section, we summarize the construction of free commutative integro-differential algebras by initialization [27].

3.1. Regular differential algebras. The construction applies to a large class of differential algebras called regular differential algebras. So we begin with the concept and examples of regular differential algebras.

\subsubsection{Quasi-antiderivatives and regularity.}

Definition 3.1. Let $(A, d)$ be a differential algebra of weight $\lambda$ with derivation $d$. A linear map $Q: A \rightarrow A$ is called a quasi-antiderivative if $d \circ Q \circ d=d$ and $Q \circ d \circ Q=Q$, with the additional condition that $\operatorname{ker} Q$ is a nonunitary $\mathbf{k}$-subalgebra of $A$ when $\lambda \neq 0$. A differential algebra whose derivation has a quasi-antiderivative is called regular.

Given a regular differential k-algebra $(A, d)$ and a fixed quasi-antiderivative $Q$ for $d$, we define the following operators. Let

$$
E=i d_{A}-Q \circ d, S=d \circ Q, J=i d_{A}-E=Q \circ d, T=i d_{A}-S .
$$

We also define $A_{J}$ to be the $\mathbf{k}$-submodule $A_{J}=i m Q$, and $A_{T}$ to be the k-subalgebra $\operatorname{ker} Q$.

Regularity is equivalent to the existence of certain projectors, namely idempotent linear maps to a subspace.

Proposition 3.2. ([27]) Let $(A, d)$ be a regular differential algebra. If $A$ is regular and $Q$ a quasi-antiderivation for $d$, then the corresponding $S:=d \circ Q: A \rightarrow A$ is a projector onto im $d$ and $E:=\mathrm{id}_{A}-Q \circ d: A \rightarrow A$ is a projector onto ker $d$. Conversely, if there are projectors $S: A \rightarrow A$ onto im $d$ and $E: A \rightarrow A$ onto $\operatorname{ker} d$, then there is a unique quasi-antiderivative $Q$ of $d$ such that $\operatorname{im} Q=\operatorname{ker} E$ and $\operatorname{ker} Q=\operatorname{ker} S$ and $(A, d)$ is regular. 
To prove the converse, from the given projectors $S$ and $E$, we have $A=\operatorname{ker} S \oplus \operatorname{im} d$ and $A=\operatorname{ker} d \oplus \operatorname{ker} E$. Thus the restriction of $d$ to ker $E$ is a bijection onto im $d$. Then there is unique map $Q: A \rightarrow A$ whose restriction to im $d$ is the inverse of the above bijection and whose kernel is $\operatorname{ker} S$.

3.1.2. Differential polynomial algebras. Let $Y$ be a set with a well-ordering $\leq_{Y}$. Define the length-lexicographic order $\leq_{Y, \text { lex }}^{*}$ on the free monoid $M(Y)$ by

$$
u<_{Y, \text { lex }}^{*} v \Leftrightarrow\left\{\begin{array}{l}
\ell<m, \\
\text { or } \ell=m \text { and } \exists 1 \leq i_{0} \leq \ell \text { such that } u_{i}=v_{i} \text { for } 1 \leq i<i_{0} \text { and } u_{i_{0}}<v_{i_{0}},
\end{array}\right.
$$

where $u=u_{1} \cdots u_{\ell}$ and $v=v_{1} \cdots v_{m}$ with $u_{i}, v_{j} \in Y, 1 \leq i \leq \ell, 1 \leq j \leq m$. It is well-known [3] that $\leq_{Y, \text { lex }}^{*}$ is again a well-ordering. An element $1 \neq u$ of the free commutative monoid $C(Y)$ can be uniquely expressed as

$$
u=u_{0}^{j_{0}} \cdots u_{k}^{j_{k}}, \text { where } u_{0}, \cdots, u_{k} \in Y, j_{0}, \cdots, j_{k} \in \mathbb{Z}_{\geq 1} \text { and } u_{0}>\cdots>u_{k} .
$$

This expression is called the standard form of $u$. If $k=-1$, we take $u=1$ by convention.

Let $X$ be a well-ordered set and let $Y=\Delta X$ (resp. $\left.\Delta_{n} X\right)$. Let $n \geq 0$ be given. For $x_{0}^{\left(i_{0}\right)}, x_{1}^{\left(i_{1}\right)} \in Y$ with $x_{0}, x_{1} \in X$, define

$$
x_{0}^{\left(i_{0}\right)} \leq x_{1}^{\left(i_{1}\right)}\left(\text { resp. } x_{0}^{\left(i_{0}\right)} \leq_{n} x_{1}^{\left(i_{1}\right)}\right) \Leftrightarrow\left(x_{0},-i_{0}\right) \leq\left(x_{1},-i_{1}\right) \quad \text { lexicographically. }
$$

For example $x^{(2)}<x^{(1)}<x$. Also, $x_{1}<x_{2}$ implies $x_{1}^{\left(i_{1}\right)}<x_{2}^{\left(i_{2}\right)}$ for all $i_{1}, i_{2} \geq 0$.

Definition 3.3. Let $u \in C(\Delta X)$ with standard form in Eq. (21):

$$
u=u_{0}^{j_{0}} \cdots u_{k}^{j_{k}} \text {, where } u_{0}, \cdots, u_{k} \in \Delta X, u_{0}>\cdots>u_{k} \text { and } j_{0}, \cdots, j_{k} \in \mathbb{Z}_{\geq 1} .
$$

Call $u$ functional if either $u=1$ or $u_{k} \in X$ or $j_{k}>1$.

Proposition 3.4. ([23, 27]) Let $\lambda \in \mathbf{k}$ and let $X$ be a set. Let $A=\left(\mathbf{k}\{X\}, d_{\lambda}\right)$ be the free commutative differential algebra of weight $\lambda$ on $X$ as defined in Theorem 2.3. (b), Then there are direct sums $A=A_{T} \oplus \operatorname{im} d$ and $A=A_{J} \oplus \operatorname{ker} d$, where

$$
\mathcal{A}_{T}=\mathcal{A}_{T, n}=\left\{u \in C\left(\Delta_{n} X\right) \mid u \text { is functional }\right\}, \quad A_{T}:=\mathbf{k} \mathcal{A}_{T},
$$

and $A_{J}$ is the submodule generated by all monomials $1 \neq u \in C(\Delta X)$. Thus $d$ admits a quasiantiderivative $Q$. Therefore, $\left(\mathbf{k}\{X\}, d_{\lambda}\right)$ is regular.

Since the product of two functional monomials is again functional, $A_{T}$ is in fact a k-subalgebra of $A$.

As noted in the remark after Proposition 3.2, the quasi-antiderivative $Q$ is defined as follows. From the direct sums, the derivation $D$ restricts to a bijection $D: A_{J} \rightarrow \operatorname{im} d$. Define $Q: \operatorname{im} d \rightarrow$ $A_{J}$ to be the inverse map and then extend $Q$ to $A$ by taking $A_{T}$ to be the kernel of $Q$.

3.1.3. Rational functions. We show that the algebra of rational functions with derivation of any weight is regular.

Let $A=\mathbb{C}(x)$. For given $\lambda \in \mathbb{C}$, let

$$
d_{\lambda}: A \rightarrow A, f(x) \mapsto \begin{cases}\frac{f(x+\lambda)-f(x)}{\lambda}, & \lambda \neq 0, \\ f^{\prime}(x), & \lambda=0,\end{cases}
$$

be the $\lambda$-derivation. Denote 


$$
\mathcal{R}:= \begin{cases}\left\{\sum_{i=1}^{k} \sum_{j=1}^{n_{i}} \frac{\gamma_{i j}}{\left(x-\alpha_{i}\right)^{j}} \mid \alpha_{i} \in \mathbb{C} \text { distinct }, \gamma_{i j} \in \mathbb{C}\right\}, & \lambda=0, \\ \left\{\sum_{i=1}^{k} \sum_{j=1}^{n_{i}} \frac{\gamma_{i j}}{\left(x-\alpha_{i j}\right)^{i}} \mid \alpha_{i j} \in \mathbb{C} \text { distinct for any given } i, \gamma_{i j} \in \mathbb{C} \text { nonzero }\right\}, & \lambda \neq 0 .\end{cases}
$$

Then denote

$$
\mathbb{C}(x)_{J}:=x \mathbb{C}[x]+\mathcal{R}
$$

and

$$
\mathbb{C}(x)_{T}:= \begin{cases}\left\{\sum_{i=1}^{k} \frac{\gamma_{i}}{x-\alpha_{i}} \mid \alpha_{i} \in \mathbb{C} \text { distinct }, \gamma_{i} \in \mathbb{C}\right\}, & \lambda=0, \\ \left\{\sum_{i=1}^{k} \sum_{j=1}^{n_{i}} \frac{\gamma_{i j}}{\left(x-\alpha_{i j}\right)^{i}} \in \mathcal{R} \mid \operatorname{re}\left(\alpha_{i j}\right) \in[0,|\operatorname{re}(\lambda)|)\right\}, & \lambda \neq 0,\end{cases}
$$

where $\operatorname{re}(z)$ is the real part of $z \in \mathbb{C}$. It is proved in [27] that

$$
\mathbb{C}(x)=\operatorname{im} d_{\lambda} \oplus \mathbb{C}(x)_{T} .
$$

Further, $\mathbb{C}(x)_{T}$ is a nonunitary subalgebra of $\mathbb{C}(x)$. We also have

$$
\mathbb{C}(x)=\operatorname{ker} d_{\lambda} \oplus \mathbb{C}(x)_{J} .
$$

Then by Proposition 3.2, $d_{\lambda}$ is regular.

3.2. Construction of $\operatorname{ID}(A)^{*}$. We now give the construction of the free commutative integrodifferential algebra $\operatorname{ID}(A)^{*}$ on a regular differential algebra $(A, d)$ with a fixed quasi-antiderivative $Q$.

With the notations set up after Definition 3.1, we give now an explicit construction of $\operatorname{ID}(A)^{*}$ via free commutative Rota-Baxter algebras and tensor products. First let

$$
\amalg_{T}(A):=\bigoplus_{k \geq 0} A \otimes A_{T}^{\otimes k}=A \oplus\left(A \otimes A_{T}\right) \oplus\left(A \otimes A_{T}^{\otimes 2}\right)+\cdots
$$

be the k-submodule of $\amalg(A)$ in Eq. (11). Then $\amalg_{T}(A)$ is the tensor product $A \otimes \amalg^{+}\left(A_{T}\right)$ where $\amalg^{+}\left(A_{T}\right):=\bigoplus_{n \geq 0} A_{T}^{\otimes n}$ is the mixable shuffle algebra [21, 24, 32] on the non-unitary k-algebra $A_{T}$.

Next, let $K:=\operatorname{ker} d \supset \mathbf{k}$ and let

$$
A_{\varepsilon}:=\{\varepsilon(a) \mid a \in A\}
$$

denote a replica of the $K$-algebra $A$, endowed with the zero derivation and the $K$-algebra structure map

$$
K \rightarrow A_{\varepsilon}, \quad c \mapsto \varepsilon(c), c \in K .
$$

We will use the $K$-algebra isomorphism

$$
\varepsilon: A \rightarrow A_{\varepsilon}, \quad a \mapsto \varepsilon(a), a \in A .
$$

Let

$$
\mathrm{ID}(A)^{*}:=A_{\varepsilon} \otimes_{K} \amalg_{T}(A)=A_{\varepsilon} \otimes_{K} A \otimes \amalg^{+}\left(A_{T}\right)
$$

denote the tensor product differential algebra of $A_{\varepsilon}$ and $\amalg_{T}(A)$, namely the tensor product algebra where the derivation $d_{A}$ is defined by the Leibniz rule. To define the linear operator $\Pi_{A}$ on $\operatorname{ID}(A)^{*}$, 
we first require that $\Pi_{A}$ be $A_{\varepsilon}$-linear. Then we just need to define $\Pi_{A}(\mathfrak{a})$ for a pure tensor $\mathfrak{a}$ in $A \otimes \amalg^{+}\left(A_{T}\right)$. For this purpose we apply induction on the length $n$ of $\mathfrak{a}$.

When $n=1$, we have $\mathfrak{a}=a \in A$. By definition of $T$ we have $a=d(Q(a))+T(a)$ with $T(a) \in A_{T}$. Then we define

$$
\Pi_{A}(a):=Q(a)-\varepsilon(Q(a))+1 \otimes T(a) .
$$

Assume that $\Pi_{A}(\mathfrak{a})$ has been defined for $\mathfrak{a}$ of length $n \geq 1$ and consider the case when $\mathfrak{a}$ has length $n+1$. Then $\mathfrak{a}=a \otimes \overline{\mathfrak{a}}$ where $a \in A, \overline{\mathfrak{a}} \in A_{T}^{\otimes n}$ and we define

$$
\Pi_{A}(a \otimes \overline{\mathfrak{a}}):=Q(a) \otimes \overline{\mathfrak{a}}-\Pi_{A}(Q(a) \overline{\mathfrak{a}})-\lambda \Pi_{A}(d(Q(a)) \overline{\mathfrak{a}})+1 \otimes T(a) \otimes \overline{\mathfrak{a}},
$$

where the first and last terms are already in $A \otimes \amalg^{+}\left(A_{T}\right)$ while the middle terms are in $\operatorname{ID}(A)^{*}$ by the induction hypothesis.

Theorem 3.5. ([27]) Let $(A, d)$ be a regular differential algebra of weight $\lambda$ with a fixed quasiantiderivative $Q$. Then the triple $\left(\operatorname{ID}(A)^{*}, d_{A}, \Pi_{A}\right)$, with the natural embedding

$$
i_{A}: A \hookrightarrow \mathrm{ID}(A)^{*}=A_{\varepsilon} \otimes_{K} A \otimes \amalg^{+}\left(A_{T}\right)
$$

onto the second tensor factor, is the free commutative integro-differential algebra of weight $\lambda$ generated by $A$.

\section{Free commutative integro-Differential algebras by Gröbner-Shirshov bases}

In this section, we give a construction of free commutative integro-differential algebras by the method of Gröbner-Shirshov bases. The main result Theorem 4.14 can be read independently of the rest of the section, which is meant to give some details of the method.

The method of Gröbner bases or Gröbner-Shirshov bases originated from the work of Buchberger [13] (for commutative polynomial algebras, 1965), Hironaka [31] (for infinite series algebras, 1964), Shirshov [43] (for Lie algebras, 1962) and Zhukov [47] (reduction in nonassociative algebra, 1950). It has since become a fundamental method in commutative algebra, algebraic geometry and computational algebra, and has been extended to many other algebraic structures, notably associative algebras [6, 7]. In recent years, the method of Gröbner-Shirshov bases has been applied to a large number of algebraic structures to study problems on normal forms, word problems, rewriting systems, embedding theorems, extensions, growth functions and Hilbert series. See [8, 10, 12] for further details.

The method of Gröbner bases or Gröbner-Shirshov bases is very useful in constructing free objects in various categories, including the alternative constructions of free Rota-Baxter algebras and free differential Rota-Baxter algebras [9, 11]. The basic idea is to prove a compositiondiamond lemma that achieves a rewriting procedure for reducing any element to a certain "standard form". Then the set of elements in standard form is a basis of the free object.

In the recent paper[23], this method is applied to construct the free commutative integrodifferential algebra as the quotient of a free commutative differential Rota-Baxter algebra modulo the integration by parts formula in Eq. (7). In order to do so, the authors first establish a Composition-Diamond Lemma for the free commutative differential Rota-Baxter algebra constructed in [26]. Then they prove that the ideal generated by the defining relation of integrodifferential algebras in Eq. (7) has a Gröbner-Shirshov basis, thereby identifying a basis of the free commutative integro-differential algebra as a canonical subset of a known basis of a free commutative differential Rota-Baxter algebra. 
4.1. Weakly monomial order. In this subsection, we will define a weak form of the monomial order on pieces of the set of differential Rota-Baxter monomials filtered by the order of differentiation. It will be sufficient to establish the composition-diamond lemma for integro-differential algebras.

For a set $X$, recall that $\Delta X:=\left\{x^{(k)} \mid x \in X, k \geq 0\right\}$ and $\Delta_{n} X:=\left\{x^{(k)} \mid x \in X, 0 \leq k \leq n\right\}$ for $n \geq 0$. Then the family $\left\{C\left(\Delta_{n} X\right)\right\}_{n \geq 0}$ defines an increasing filtration on $C(\Delta X)$ and hence by Eq. (14), induces a filtration $\left\{\mathcal{B}\left(\Delta_{n} X\right)\right\}_{n \geq 0}$ of the set $\mathcal{B}(\Delta X)$ of DRB monomials by DRb monomials of order $n$. Elements of $\mathcal{B}\left(\Delta_{n} X\right)$ are called DRB monomials of order $n$.

In Definition 4.1 below and what follows, the DRB ( $\star$-DRB) monomials are elements in the basis $\mathcal{B}(\Delta X)\left(\right.$ resp. $\left.\mathcal{B}\left(\Delta X^{\star}\right)\right)$ of $\amalg(\mathbf{k}\{X\})$ (resp. $\left.\amalg\left(\mathbf{k}\left\{X^{\star}\right\}\right)\right)$, which are identified via Eq. (18) as $(d, P)$-bracketed monomials $\eta(\mathcal{B}(\Delta X)) \subseteq \mathbf{k} \mathscr{C}(\Delta X)\left(\operatorname{resp} . \eta\left(\mathcal{B}\left(\Delta X^{\star}\right)\right) \subseteq \mathbf{k} \mathscr{C}\left(\Delta X^{\star}\right)\right)$.

Definition 4.1. Let $X$ be a set, $\star$ a symbol not in $X$ and $\Delta_{n} X^{\star}:=\Delta_{n}(X \cup\{\star\})$.

(a) By a $\star$-DRB monomial on $\Delta_{n} X$, we mean any expression in $\mathcal{B}\left(\Delta_{n} X^{\star}\right)$ with exactly one occurrence of $\star$. The set of all $\star$-DRB monomials on $\Delta_{n} X$ is denoted by $\mathcal{B}^{\star}\left(\Delta_{n} X\right)$.

(b) For $q \in \mathcal{B}^{\star}\left(\Delta_{n} X\right)$ and $u \in \mathcal{B}\left(\Delta_{n} X\right)$, we define

$$
\left.q\right|_{u}:=\left.q\right|_{\star \mapsto u}
$$

to be the bracketed monomial in $\mathfrak{C}\left(\Delta_{n} X\right)$ obtained by replacing the letter $\star$ in $q$ by $u$, and call $\left.q\right|_{u}$ a $u$-monomial on $\Delta_{n} X$.

(c) Further, for $s=\sum_{i} c_{i} u_{i} \in \mathbf{k} \mathcal{B}\left(\Delta_{n} X\right)$, where $c_{i} \in \mathbf{k}, u_{i} \in \mathcal{B}\left(\Delta_{n} X\right)$ and $q \in \mathcal{B}^{\star}\left(\Delta_{n} X\right)$, we define

$$
\left.q\right|_{s}:=\left.\sum_{i} c_{i} q\right|_{u_{i}}
$$

which is in $\mathbf{k} \mathfrak{C}\left(\Delta_{n} X\right)$.

We note that a $\star$-DRB monomial $q$ is a DRB monomial in $\Delta_{n} X^{\star}$ while its substitution $\left.q\right|_{u}$ might not be a DRB monomials. For example, for $q=P\left(x_{1}\right) \star \in \eta\left(\mathcal{B}\left(\Delta_{n} X^{\star}\right)\right)$ and $u=P\left(x_{2}\right) \in \mathcal{B}\left(\Delta_{n} X\right)$ where $x_{1}, x_{2} \in X$, the $u$-monomial $\left.q\right|_{u}=P\left(x_{1}\right) P\left(x_{2}\right)$ is no longer in $\eta\left(\mathcal{B}\left(\Delta_{n} X\right)\right)$.

Definition 4.2. If $q=\left.p\right|_{d^{\ell}(\star)}$ for some $p \in \mathcal{B}^{\star}\left(\Delta_{n} X\right)$ and $\ell \in \mathbb{Z}_{\geq 1}$, then we call $q$ a type I $\star$-DRB monomial. Let $\mathcal{B}_{\mathrm{I}}^{\star}\left(\Delta_{n} X\right)$ denote the set of type I $\star$-DRB monomials on $\Delta_{n} X$ and call

$$
\mathcal{B}_{\text {II }}^{\star}\left(\Delta_{n} X\right):=\mathcal{B}^{\star}\left(\Delta_{n} X\right) \backslash \mathcal{B}_{\mathrm{I}}^{\star}\left(\Delta_{n} X\right)
$$

the set of type II $\star$-DRB monomials.

For example, $d(\star) P(x) \in \mathcal{B}_{\mathrm{I}}^{\star}\left(\Delta_{n} X\right)$ and $\star P(x) \in \mathcal{B}_{\mathrm{II}}^{\star}\left(\Delta_{n} X\right)$.

Definition 4.3. Let $X$ be a set, $\star_{1}, \star_{2}$ two distinct symbols not in $X$ and $\Delta_{n} X^{\star_{1}, \star_{2}}:=\Delta_{n}(X \cup$ $\left.\left\{\star_{1}, \star_{2}\right\}\right)$. We define a $\left(\star_{1}, \star_{2}\right)$-DRB monomial on $\Delta_{n} X$ to be an expression in $\mathcal{B}\left(\Delta_{n} X^{\star_{1}, \star_{2}}\right)$ with exactly one occurrence of $\star_{1}$ and exactly one occurrence of $\star_{2}$. The set of all $\left(\star_{1}, \star_{2}\right)$-DRB monomials on $\Delta_{n} X$ is denoted by $\mathcal{B}^{\star_{1}, \star_{2}}\left(\Delta_{n} X\right)$. For $q \in \mathcal{B}^{\star_{1}, \star_{2}}\left(\Delta_{n} X\right)$ and $u_{1}, u_{2} \in \mathbf{k} \mathcal{B}\left(\Delta_{n} X\right)$, we define

$$
\left.q\right|_{u_{1}, u_{2}}:=\left.q\right|_{\star_{1} \mapsto u_{1}, \star_{2} \mapsto u_{2}}
$$

to be the bracketed monomial obtained by replacing the letter $\star_{1}\left(\right.$ resp. $\left.\star_{2}\right)$ in $q$ by $u_{1}\left(\right.$ resp. $\left.u_{2}\right)$ and call it a $\left(u_{1}, u_{2}\right)$-bracketed monomial on $\Delta_{n} X$.

A $\left(u_{1}, u_{2}\right)$-DRB monomial on $\Delta_{n} X$ can also be recursively defined by

$$
\left.q\right|_{u_{1}, u_{2}}:=\left.\left(\left.q^{\star}\right|_{u_{1}}\right)\right|_{u_{2}}
$$


where $q^{\star_{1}}$ is $q$ when $q$ is regarded as a $\star_{1}$-DRB monomial on the set $\Delta_{n} X^{\star_{2}}$. Then $\left.q^{\star_{1}}\right|_{u_{1}}$ is in $\mathcal{B}^{\star 2}\left(\Delta_{n} X\right)$. Similarly, we have

$$
\left.q\right|_{u_{1}, u_{2}}:=\left.\left(\left.q^{\star_{2}}\right|_{u_{2}}\right)\right|_{u_{1}}
$$

Let $X$ be a well-ordered set. Let $n \geq 0$ be given. We extend the well-ordering $\leq_{n}$ on $C\left(\Delta_{n} X\right)$ defined in Eq. (22) to $\mathcal{B}\left(\Delta_{n} X\right)$. Note that

$$
\mathcal{B}\left(\Delta_{n} X\right)=\left\{u_{0} \otimes u_{1} \otimes \cdots \otimes u_{k} \mid u_{i} \in C\left(\Delta_{n} X\right), 1 \leq i \leq k, k \geq 0\right\}=\sqcup_{k \geq 1} C\left(\Delta_{n} X\right)^{\otimes k}
$$

can be identified with the free semigroup on the set $C\left(\Delta_{n} X\right)$. Thus the well-ordering $\leq_{n}$ on $C\left(\Delta_{n} X\right)$ extends to a well-ordering $\leq_{n \text {,lex }}^{*}$ [3] on $\mathcal{B}\left(\Delta_{n} X\right)$ which we will still denote by $\leq_{n}$ for simplicity. More precisely, for any $u=u_{0} \otimes \cdots \otimes u_{k} \in C\left(\Delta_{n} X\right)^{\otimes(k+1)}$ and $v=v_{0} \otimes \cdots \otimes v_{\ell} \in C\left(\Delta_{n} X\right)^{\otimes(\ell+1)}$, define

$$
u \leq_{n} v \text { if }\left(k, u_{0}, \cdots, u_{k}\right) \leq\left(\ell, v_{0}, \cdots, v_{\ell}\right) \text { lexicographically. }
$$

Definition 4.4. Let $\leq_{n}$ be the well-ordering on $\mathcal{B}\left(\Delta_{n} X\right)$ defined in Eq. (28). Let $q \in \mathcal{B}^{\star}\left(\Delta_{n} X\right)$ and $s \in \mathbf{k} \mathcal{B}\left(\Delta_{n} X\right)$.

(a) For any $0 \neq f \in \mathbf{k} \mathcal{B}\left(\Delta_{n} X\right)$, let $\bar{f}$ denote the leading term of $f: f=c \bar{f}+\sum_{i} c_{i} u_{i}$, where $0 \neq c, c_{i} \in \mathbf{k}, u_{i} \in \mathcal{B}\left(\Delta_{n} X\right), u_{i}<\bar{f}$. We call $f$ monic if $c=1$.

(b) Let

$$
\overline{\left.q\right|_{s}}:=\overline{\operatorname{Red}\left(\left.q\right|_{s}\right)}
$$

where Red: $\mathbf{k} \mathfrak{C}\left(\Delta_{n} X\right) \rightarrow \amalg\left(\Delta_{n} X\right)=\eta\left(\mathbf{k} \mathcal{B}\left(\Delta_{n} X\right)\right)$ is the reduction map in Eq. (19).

(c) The element $\left.q\right|_{s} \in \mathbf{k} \mathfrak{C}\left(\Delta_{n} X\right)$ is called normal if $\left.q\right|_{s}$ is in $\mathcal{B}\left(\Delta_{n} X\right)$. In other words, if $\operatorname{Red}\left(\left.q\right|_{\bar{s}}\right)=\left.q\right|_{\bar{s}}$.

Remark 4.5. By definition, $\left.q\right|_{s}$ is normal if and only if $\left.q\right|_{s}$ is normal if and only if the $\bar{s}$-DRB monomial $\left.q\right|_{s}$ is already a DRB monomial, that is, no further reduction in $\amalg\left(\Delta_{n} X\right)$ is possible.

Here are some examples of abnormal $s$-DRB monomials.

Example 4.6. (a) $q=\star P(y)$ and $\bar{s}=P(x)$, giving $\left.q\right|_{\bar{s}}=P(x) P(y)$ which is reduced to $P(x P(y))+P(P(x) y)+\lambda P(x y)$ in $\eta\left(\amalg\left(\Delta_{n} X\right)\right)$

(b) $q=d(\star)$ and $\bar{s}=P(x)$, giving $\left.q\right|_{\bar{s}}=d(P(x))$ which is reduced to $x$ in $\eta\left(\amalg\left(\Delta_{n} X\right)\right)$;

(c) $q=d(\star)$ and $\bar{s}=x^{2}$, giving $\left.q\right|_{\bar{s}}=d\left(x^{2}\right)$ which is reduced to $2 x x^{(1)}+\lambda\left(x^{(1)}\right)^{2}$ in $\eta\left(\amalg\left(\Delta_{n} X\right)\right)$;

(d) $q=d^{n}(\star)$ and $\bar{s}=d(x)$, giving $\left.q\right|_{\bar{s}}=d^{n+1}(s)$ which is reduced to 0 in $\eta\left(\amalg\left(\Delta_{n} X\right)\right)$.

Definition 4.7. A weakly monomial order on $\mathcal{B}\left(\Delta_{n} X\right)$ is a well-ordering $\leq$ satisfying the following condition:

For $u, v \in \mathcal{B}\left(\Delta_{n} X\right)$, if $u \leq v$, then $\overline{\left.q\right|_{u}} \leq \overline{\left.q\right|_{v}}$ if $q \in \mathcal{B}_{\mathrm{II}}^{\star}\left(\Delta_{n} X\right)$, or if $q \in \mathcal{B}_{\mathrm{I}}^{\star}\left(\Delta_{n} X\right)$ and $\left.q\right|_{v}$ is normal.

Proposition 4.8. ([23]) The order $\leq_{n}$ defined in Eq. (28) is a weakly monomial order on $\mathcal{B}\left(\Delta_{n} X\right)$.

4.2. Composition-Diamond lemma. In this section, we shall establish the composition-diamond lemma for the free commutative differential Rota-Baxter algebra $\amalg\left(\mathbf{k}\left[\Delta_{n} X\right]\right)$ of order $n$.

Definition 4.9. (a) Let $u, w \in \mathcal{B}\left(\Delta_{n} X\right)$. We call $u$ a subword of $w$ if $w$ is in the operated ideal of $\mathfrak{C}\left(\Delta_{n} X\right)$ generated by $u$. In terms of $\star$-words, $u$ is a subword of $w$ if there is a $q \in \mathcal{B}^{\star}\left(\Delta_{n} X\right)$ such that $w=\left.q\right|_{u}$. 
(b) Let $u_{1}$ and $u_{2}$ be two subwords of $w$. Then $u_{1}$ and $u_{2}$ are called separated if $u_{1} \in C\left(\Delta_{n} X\right)$, $u_{2} \in \mathcal{B}\left(\Delta_{n} X\right)$ and there is a $q \in \mathcal{B}^{\star_{1}, \star_{2}}\left(\Delta_{n} X\right)$ such that $w=\left.q\right|_{u_{1}, u_{2}}$.

(c) For any $u \in \mathcal{B}\left(\Delta_{n} X\right), u$ can be expressed as $u=u_{1} \cdots u_{k}$, where $u_{1}, \cdots, u_{k-1} \in \Delta_{n} X$ and $u_{k} \in \Delta_{n} X \cup P\left(\mathcal{B}\left(\Delta_{n} X\right)\right)$. The expression is unique up to permutations of those factors in $\Delta_{n} X$. The integer $k$ is called the breadth of $u$ and is denoted by bre $(u)$.

(d) Let $f, g \in \mathcal{B}\left(\Delta_{n} X\right)$. A pair $(u, v)$ with $u \in \mathcal{B}\left(\Delta_{n} X\right)$ and $v \in C\left(\Delta_{n} X\right)$ is called an intersection pair for $(f, g)$ if the differential Rota-Baxter monomial $w:=f u$ equals $v g$ and satisfies $\operatorname{bre}(w)<\operatorname{bre}(f)+\operatorname{bre}(g)$. Then we call $f$ and $g$ to be overlapping. Note that if $f$ and $g$ are overlapping, then $f \in C\left(\Delta_{n} X\right)$.

For example, let $w=x y x y$ with $x, y \in X$ and $u_{1}=x y$ be the subword of $w$ on the left and $u_{1}=x y$ be the subword of $w$ on the right. Then $u_{1}$ and $u_{2}$ are separated. Let $g$ be the subword $y x$ of $w$. Then $u_{1}$ and $g$ are overlapping. A systematic discussion on relative locations (separated, overlapping and inclusion) of two subwords can be found in [22, 30].

There are three kinds of compositions.

Definition 4.10. Let $\leq_{n}$ be the weakly monomial order on $\mathcal{B}\left(\Delta_{n} X\right)$ defined in Eq. (28), and let $f, g \in \mathbf{k} \mathcal{B}\left(\Delta_{n} X\right)$ be monic with respect to $\leq_{n}$ such that $f \neq g$.

(a) If $\bar{f} \in C\left(\Delta_{n} X\right) P\left(\mathcal{B}\left(\Delta_{n} X\right)\right)$, then define a composition of multiplication to be $f u$ where $u \in C\left(\Delta_{n} X\right) P\left(\mathcal{B}\left(\Delta_{n} X\right)\right)$.

(b) If there is an intersection pair $(u, v)$ for $(\bar{f}, \bar{g})$, then we define

$$
(f, g)_{w}:=(f, g)_{w}^{u, v}:=f u-v g
$$

and call it an intersection composition of $f$ and $g$.

(c) If there exists a $q \in \mathcal{B}^{\star}\left(\Delta_{n} X\right)$ such that $w:=\bar{f}=\left.q\right|_{\bar{g}}$, then we define $(f, g)_{w}:=(f, g)_{w}^{q}:=$ $f-\left.q\right|_{g}$ and call it an inclusion composition of $f$ and $g$ with respect to $q$. Note that if this is the case, then $\left.q\right|_{g}$ is normal.

In the last two cases, $(f, g)_{w}$ is called the ambiguity of the composition. For example, let

$$
f=P(d(u) P(d(v) P(r)))-u P(d(v) P(r))+P(u d(v) P(r))+\lambda P(d(u) d(v) P(r))
$$

and

$$
g=P(d(v) P(r))-v P(r)+P(v r)+\lambda P(d(v) r)
$$

with the first terms being the leading terms. Then we have $\bar{f}=\left.q\right|_{\bar{g}}$ where $q:=P(d(u) \star)$. Hence we get an inclusion composition of $f$ and $g$ with the ambiguity

$$
\begin{aligned}
(f, g)_{w}^{q}= & -u P(d(v) P(r))+P(u d(v) P(r))+\lambda P(d(u) d(v) P(r)) \\
& -(-P(d(u) v P(r))+P(d(u) P(v r))+\lambda P(d(u) P(d(v) r))) .
\end{aligned}
$$

Definition 4.11. Let $\leq_{n}$ be the weakly monomial order on $\mathcal{B}\left(\Delta_{n} X\right)$ defined in Eq. (28), $S \subseteq$ $\mathbf{k} \mathcal{B}\left(\Delta_{n} X\right)$ be a set of monic differential Rota-Baxter polynomials and $w \in \mathcal{B}\left(\Delta_{n} X\right)$.

(a) A composition of multiplication $f u$ is called trivial mod $[S]$ if

$$
f u=\left.\sum_{i} c_{i} q_{i}\right|_{s_{i}}
$$

where $c_{i} \in \mathbf{k}, q_{i} \in \mathcal{B}^{\star}\left(\Delta_{n} X\right), s_{i} \in S,\left.q_{i}\right|_{s_{i}}$ is normal and $\left.q_{i}\right|_{\overline{s_{i}}} \leq_{n} \overline{f u}$. If this is the case, we write

$$
f u \equiv 0 \bmod [S]
$$


(b) For $u, v \in \mathbf{k} \mathcal{B}\left(\Delta_{n} X\right)$ and $w \in \mathcal{B}\left(\Delta_{n} X\right)$, we say $u$ and $v$ are congruent modulo $(S$, w) and denote this by

$$
u \equiv v \bmod (S, w)
$$

if $u-v=\left.\sum_{i} c_{i} q_{i}\right|_{s_{i}}$, where $c_{i} \in \mathbf{k}, q_{i} \in \mathcal{B}^{\star}\left(\Delta_{n} X\right), s_{i} \in S,\left.q_{i}\right|_{s_{i}}$ is normal and $\overline{\left.q_{i}\right|_{s_{i}}}<_{n} w$.

(c) For $f, g \in \mathbf{k} \mathcal{B}\left(\Delta_{n} X\right)$ and suitable $u, v$ or $q$ that give an intersection composition $(f, g)_{w}^{u, v}$ or an inclusion composition $(f, g)_{w}^{q}$, the composition is called trivial modulo $(S, w)$ if

$$
(f, g)_{w}^{u, v} \text { or }(f, g)_{w}^{q} \equiv 0 \bmod (S, w) .
$$

(d) The set $S \subseteq \mathbf{k} \mathcal{B}\left(\Delta_{n} X\right)$ is a Gröbner-Shirshov basis if all compositions of multiplication are trivial $\bmod [S]$, and, for $f, g \in S$, all intersection compositions $(f, g)_{w}^{u, v}$ and all inclusion compositions $(f, g)_{w}^{q}$ are trivial modulo $(S, w)$.

Theorem 4.12. ([23] Composition-Diamond Lemma) Let $\leq_{n}$ be the weakly monomial order on $\mathcal{B}\left(\Delta_{n} X\right)$ defined in Eq. (28), $S_{n}$ a set of monic DRB polynomials in $\mathbf{k} \mathcal{B}\left(\Delta_{n} X\right)$ with $d\left(S_{n}\right) \subseteq S_{n}$, and $\operatorname{Id}\left(S_{n}\right)$ the Rota-Baxter ideal of $\mathbf{k} \mathcal{B}\left(\Delta_{n} X\right)$ generated by $S_{n}$. Then with respect to $\leq_{n}$, the following conditions are equivalent:

(a) $S_{n}$ is a Gröbner-Shirshov basis in $\mathbf{k} \mathcal{B}\left(\Delta_{n} X\right)$.

(b) If $0 \neq f \in \operatorname{Id}\left(S_{n}\right)$, then $\bar{f}=\left.q\right|_{s}$ for some $q \in \mathcal{B}^{\star}\left(\Delta_{n} X\right)$, $s \in S_{n}$ and $\left.q\right|_{s}$ is normal.

(c) The set $\operatorname{Irr}\left(S_{n}\right):=\mathcal{B}\left(\Delta_{n} X\right) \backslash\left\{\left.q\right|_{s}\left|q \in \mathcal{B}^{\star}\left(\Delta_{n} X\right), s \in S_{n}, q\right|_{s}\right.$ is normal $\}$ is a $\mathbf{k}$-basis of $\mathbf{k} \mathcal{B}\left(\Delta_{n} X\right) / \operatorname{Id}\left(S_{n}\right)$. In other words, $\mathbf{k} \operatorname{Irr}\left(S_{n}\right) \oplus \operatorname{Id}\left(S_{n}\right)=\mathbf{k} \mathcal{B}\left(\Delta_{n} X\right)$.

4.3. Free commutative integro-differential algebras by Gröbner-Shirshov bases. In this subsection we begin with a finite set $X$ and prove that the relation ideal of the free commutative differential Rota-Baxter algebra on $X$ of order $n \geq 1$, defining the corresponding commutative integro-differential algebra of order $n$ possesses a Gröbner-Shirshov basis. Then we apply the Composition-Diamond Lemma in Theorem 4.12 to construct a canonical basis for the free commutative integro-differential algebra of order $n$. As $n$ approaches infinity, we obtain a canonical basis of the free commutative integro-differential algebra on the finite set $X$. Finally for any wellordered set $X$, by showing that the canonical basis of the free commutative integro-differential algebra on each finite subset of $X$ is compatible with the inclusion of the subset in $X$, we obtain a canonical basis of the free commutative integro-differential algebra on $X$.

Theorem 4.13. ([23]) Let

$$
S_{n}:=\left\{P(d(u) P(v))-u P(v)+P(u v)+\lambda P(d(u) v) \mid u, v \in \amalg\left(\mathbf{k}\left[\Delta_{n} X\right]\right)\right\}
$$

be the set of generators corresponding to the integration by parts axiom Eq. (囚). Let $\leq_{n}$ be the monomial order defined in Eq. (28).

(a) With respect to $\leq_{n}, S_{n}$ is a Gröbner-Shirshov basis in $\mathrm{W}\left(\mathbf{k}\left[\Delta_{n} X\right]\right)$. Hence $\operatorname{Irr}\left(S_{n}\right)$ in Theorem 4.12 is a linear basis of $\mathrm{W}\left(\mathbf{k}\left[\Delta_{n} X\right]\right) / \operatorname{Id}\left(S_{n}\right)$.

(b) Let $A_{T}=\mathbf{k}\{X\}_{T}$ be as defined in Eq. (23), $A_{n}=\mathbf{k}\left[\Delta_{n} X\right], A_{n, T}=A_{n} \cap A_{T}$. Let $I_{\mathrm{ID}, n}$ be the differential Rota-Baxter ideal of $\amalg\left(A_{n}\right)$ generated by $S_{n}$. Then we have the isomorphism of modules

$$
\amalg\left(A_{n}\right) / I_{I D, n} \cong A_{n} \oplus\left(\bigoplus_{k \geq 0} A_{n} \otimes A_{n-1, T}^{\otimes k} \otimes A_{n}\right) .
$$


Let

$$
S:=\{P(d(u) P(v))-u P(v)+P(u v)+\lambda P(d(u) v) \mid u, v \in \amalg(\Delta X)\} .
$$

be the set of generators corresponding to the integration by parts axiom Eq. (7).

Theorem 4.14. ([23]) Let $X$ be a nonempty well-ordered set, $A_{T}=\mathbf{k}\{X\}_{T}, \amalg(\mathbf{k}\{X\})=\amalg(\Delta X)$ the free commutative differential Rota-Baxter algebra on $X$ and $I_{I D}$ the ideal of $\amalg(\mathbf{k}\{X\})$ generated by $S$ defined in Eq. (30). Then the composition

$$
\amalg(A)_{T}:=A \oplus\left(\bigoplus_{k \geq 0} A \otimes A_{T}^{\otimes k} \otimes A\right) \hookrightarrow \amalg(A) \rightarrow \amalg(A) / I_{I D}
$$

of the inclusion and the quotient map is an isomorphism of $\mathbf{k}$-modules.

It would be interesting to compare the two constructions of free commutative integro-differential algebras in Theorem 3.5 and Theorem 4.14. The advantages of the first construction is that it applies to a large class of differential algebras and that the product in the free algebra is clearly defined. The advantage of the second construction is that the construction comes from a subset of the free commutative differential Rota-Baxter algebra from which the free integro-differential algebra is obtained modulo an ideal. It is useful to have both of the two constructions available in order to study different aspects of free commutative integro-differential algebras. Further study in this direction is being pursued in another work. The construction of free noncommutative integro-differential algebras is also under investigation.

Acknowledgements: This work is supported by the National Natural Science Foundation of China (Grant No. 11201201 and 11371178), Fundamental Research Funds for the Central Universities (Grant No. lzujbky-2013-8), the Natural Science Foundation of Gansu Province (Grant No. 1308RJZA112) and the National Science Foundation of US (Grant No. DMS 1001855). The authors thank the referees and editors for helpful comments.

\section{REFERENCES}

[1] H. Albrecher, C. Constantinescu, G. Pirsic, G. Regensburger, and M. Rosenkranz, An algebraic operator approach to the analysis of Gerber-Shiu functions, Insurance Math. Econom. 46 (2010), 42-51. 2

[2] H. Albrecher, C. Constantinescu, Z. Palmowski, G. Regensburger, and M. Rosenkranz, Exact and asymptotic results for insurance risk models with surplus-dependent premiums, SIAM J. Appl. Math. 73 (2013), 47-66. 2

[3] F. Baader T. and Nipkow, Term Rewriting and All That, Cambridge U. P., Cambridge, 1998. 7, 9, 13,

[4] C. Bai, A unified algebraic approach to classical Yang-Baxter equation, J. Phys. A 40 (2007), 1107311082. 2

[5] G. Baxter, An analytic problem whose solution follows from a simple algebraic identity, Pacific J. Math. 10 (1960), 731-742. 目

[6] G. M. Bergman, The diamond lemma for ring theory, Adv. Math. 29 (1978), 178-218. 11

[7] L. A. Bokut, Imbeddings into simple associative algebras, Algebra i Logika 15 (1976), 117-142. 11

[8] L. A. Bokut, Y. Chen, Y. Chen, Composition-Diamond lemma for tensor product of free algebras, J. Algebra 323 (2010), 2520-2537. 11

[9] L. A. Bokut, Y. Chen and X. Deng, Gröbner-Shirshov bases for Rota-Baxter algebras, Siberian Math. J. 51 (2010), 978-988. 11

[10] L. A. Bokut, Y. Chen and Y. Li, Gröbner-Shirshov bases for categories, in "Operads and Universal Algebra", World Scientific Press, (2012) 1-23. 11

[11] L. A. Bokut, Y. Chen and J. Qiu, Gröbner-Shirshov bases for associative algebras with multiple operators and free Rota-Baxter algebras, J. Pure Appl. Algebra 214 (2010) 89-110. 6, 11 
[12] L.A. Bokut, V. Latyshev, I. Shestakov and E. Zelmanov (Eds.), Selected Works of A.I. Shirshov, Birkhäuser, Basel, Boston, Berlin, 2009, vii, 241 pp. transl. M. Bremner, M. Kotchetov. 11

[13] B. Buchberger, An algorithm for finding a basis for the residue class ring of a zero-dimensional polynomial ideal [in German], Ph.D. thesis, University of Innsbruck, Austria, (1965). 11

[14] P. Cartier, On the structure of free Baxter algebras, Adv. Math. 9 (1972), 253-265.

[15] P. Cassidy, L.Guo, W. Keigher and W. Sit (Eds.), Differential Algebra and Related Topics (Proceedings for the International Workshop in Newark, NJ, 2000), World Sci. Publishing, 2002.

[16] A. Connes and D. Kreimer, Renormalization in quantum field theory and the Riemann-Hilbert problem. I. The Hopf algebra structure of graphs and the main theorem, Comm. Math. Phys., 210, (2000), no. 1, 249-273.2

[17] P. M. Cohn, Algebra, Vol.3, 2nd ed., J. Wiley \& Sons, Chichester, 1991.7

[18] K. Ebrahimi-Fard and L. Guo, Mixable shuffles, quasi-shuffles and Hopf algebras, J. Algebraic Combinatorics, 24 (2006), 83-101.5

[19] K. Ebrahimi-Fard, L. Guo and D. Kreimer, Spitzer's identity and the algebraic Birkhoff decomposition in pQFT, J. Phys. A: Math. Gen. 37 (2004), 11037-11052. 目

[20] L. Guo, Operated semigroups, Motzkin paths and rooted trees, J. Algebraic Combin. 29 (2009), 35-62. 0

[21] L. Guo, Introduction to Rota-Baxter Algebra, Higher Education Press and International Press, 2012. E 4. $5,6,10$

[22] Rota-Baxter type operators, rewriting systems and Gröbner-Shirshov bases, preprint. 14

[23] X. Gao, L. Guo and S. Zheng, Construction of free commutative integro-differential algebras by the method of Gröbner-Shirshov bases, to appear in J. Algebra and Its Application, arXiv:1302.0041. 6, 已, 目11, 13, 15,10

[24] L. Guo and W. Keigher, Baxter algebras and shuffle products, Adv. Math., 150 (2000), 117-149. 2, 1, 10

[25] L. Guo and W. Keigher, On free Baxter algebras: completions and the internal construction, Adv. Math. 151 (2000), 101-127.

[26] L. Guo and W. Keigher, On differential Rota-Baxter algebras, J. Pure Appl. Algebra, 212 (2008), 522540. 2, 田6, 11

[27] L. Guo, G. Regensburger and M. Rosenkranz, On integro-differential algebras, J. Pure Appl. Algebra 218 (2014) 456-471, arXiv:1212.0266. 日, 3, 4, 8, 9, 10, 11

[28] L. Guo, W. Sit and R. Zhang, Differential type operators and Gröbner-Shirshov bases, J. Symolic Comput. (2012). 6

[29] L. Guo and B. Zhang, Renormalization of multiple zeta values, J. Algebra, 319 (2008), 3770-3809.2, 5

[30] L. Guo and S. Zheng, Relative locations of subwords in free operated semigroups, preprint. 14

[31] H. Hironaka, Resolution of singulatities of an algebraic variety over a field if characteristic zero, I, Ann. Math. 79 (1964), 109-203.11

[32] M. Hoffman, Quasi-shuffle products, J. Algebraic Combin., 11 (2000), 49-68. 5, 10

[33] E. R. Kolchin, Differential Algebras and Algebraic Groups, Academic Press, New York, 1973.⿴囗口

[34] J. Qiu, Gröbner-Shirshov bases for commutative algebras with multiple operators and free commutative Rota-Baxter algebras, Asian-European Jour. Math., to appear. 目

[35] J.F. Ritt, Differential Equations from the Algebraic Standpoint, Amer. Math. Sco. Colloq. Pub. 14, Amer. Math. Soc., New York (1934). 2

[36] J.F. Ritt, Differential Algebra, Amer. Math. Sco. Colloq. Pub. 33, Amer. Math. Soc., New York (1950). 目

[37] M. Rosenkranz and G. Regensburger, Solving and factoring boundary problems for linear ordinary differential equations in differential algebra, J. Symbolic Comput. 43 (2008), 515-544. 目

[38] M. Rosenkranz, G. Regensburger, L. Tec, and B. Buchberger, A Symbolic Framework for Operations on Linear Boundary Problems. In V.P. Gerdt, E.W. Mayr, E.H. Vorozhtsov (eds.), Computer Algebra in Scientific Computing. Proceedings of the 11th International Workshop (CASC 2009), Springer LNCS 5743, 2009, 269-283. 目

[39] M. Rosenkranz, G. Regensburger, L. Tec, and B. Buchberger, Symbolic analysis for boundary problems: From rewriting to parametrized Gröbner bases. In U. Langer and P. Paule (eds.), Numerical and Symbolic Scientific Computing: Progress and Prospects, Springer Vienna, 2012, 273-331.2

[40] G.-C. Rota, Baxter algebras and combinatorial identities I, II, Bull. Amer. Math. Soc. 75 (1969), 325329, 330-334. 目 
[41] G.-C. Rota, Baxter operators, an introduction, In: "Gian-Carlo Rota on Combinatorics, Introductory papers and commentaries”, Joseph P. S. Kung, Editor, Birkhäuser, Boston, 1995. 2 .

[42] M. A. Semenov-Tian-Shansky, What is a classical $r$-matrix?, Funct. Ana. Appl., 17 (1983) 259-272. 目

[43] A. I. Shirshov, Some algorithmic problem for $\AA$-algebras, Sibirsk. Mat. Z., 3(1962), 132-137. 11

[44] M. Singer and M. van der Put, Galois Theory of Linear Differential Equations, Springer, 2003.2

[45] Wen-Tsun Wu, On the Decision Problem and the Mechanization of Theorem Proving in Elementary Geometry. Scientia Sinica 21 (2), (1978), 159-172. Also reprinted in Contemporary Mathematics, 29 (1984), 213 - 241. 目

[46] Wen-Tsun Wu, A constructive theory of differential algebraic geometry based on works of J. F. Ritt with particular applications to mechanical theorem-proving of differential geometries, Differential Geometry and Differential Equations (Shanghai, 1985), Lecture Notes in Math. 1255, Springer-Verlag, Berlin-New York, 1987, 173-189.2

[47] A. I. Zhukov, Reduced systems of defining relations in non-associative algebras, Mat. Sb. (N.S.), 27(69) (1950), 267-280. 11

Department of Mathematics, Lanzhou University, Lanzhou, Gansu 730000, China

E-mail address: gaoxing@lzu.edu.cn

Department of Mathematics and Computer Science, Rutgers University, Newark, NJ 07102, USA

E-mail address: liguo@rutgers.edu 